\title{
ANÁlisis DE LOS MODOS DE FALLO DEL SISTEMA DE GESTIÓN DE ENERGÍA DE UN CUBESAT
}

\section{Analysis of the failure modes of the energy management system of a Cubesat}

\author{
Lía Hidalgo de los Santosa, Diorys del Rosario Peñalób, \\ Miguel Euclides Aybar Mejíace, Edwin Sánchez ${ }^{d}$ \\ y Deyslen Mariano-Hernández
}

Recibido: 5/7/2021 • Aprobado: 1/10/2021

Cómo citar: Aybar Mejía, M., Hidalgo de los Santos, L., del Rosario Peñaló, D., Sánchez, E., \& MarianoHernández, D. (2021). Análisis de los modos de fallo del sistema de gestión de energía de un Cubesat. Ciencia, Ingenierías y Aplicaciones, 4(2), 5-20. Doi: https://doi.org/10.22206/cyap.2021.v4i2.pp5-20

\section{Resumen}

Un satélite es un dispositivo que se sitúa en órbita alrededor de la Tierra con fines cientificos, militares, educacionales o de comunicaciones. Se pueden clasificar según su peso como: satélites grandes, que pesan más de 1,000 kilogramos (kg), satélites medianos, que pesan entre $500-1,000 \mathrm{~kg}, y$ satélites pequeños, que pesan menos de $500 \mathrm{~kg}$. Dentro de los satélites pequeños se encuentran los nanosatélites, los cuales pesan entre 1-10 kg. La principal ventaja que poseen los nanosatélites es que son capaces de realizar misiones complejas a un menor costo. Dentro de las limitaciones que estos presentan es que tienen poca capacidad para captar y almacenar energía debido a su tamaño. Considerando la importancia del sistema de energía durante el funcionamiento de los satélites, este artículo analiza los modos de fallos que se presentan en el sistema y cómo estos afectan a los sistemas de generación

a Área de Ingeniería, Instituto Tecnológico de Santo Domingo (INTEC). ORCID: 0000-0001-9281-5407, Correo-e: 1074194@est.intec.edu.do b Área de Ingeniería, Instituto Tecnológico de Santo Domingo (INTEC). ORCID: 0000-0002-8533-0601, Correo-e: 1076866@est.intec.edu.do c Área de Ingeniería, Instituto Tecnológico de Santo Domingo (INTEC). ORCID: 0000-0002-4715-3499, Correo-e: miguel.aybar@.intec.edu.do d Área de Ingeniería, Instituto Tecnológico de Santo Domingo (INTEC). ORCID: 0000-0002-8592-8224, Correo-e: edwin.sanchez@intec.edu.do e Área de Ingeniería, Instituto Tecnológico de Santo Domingo (INTEC). ORCID: 0000-0002-4255-3450, Correo-e: deyslen.mariano@intec.edu.do 
y almacenamiento de energía. Para la simulación de estos sistemas se determinaron la cantidad minima de paneles solares y baterías necesarios para su correcto funcionamiento. Los resultados obtenidos muestran que con este análisis se logra dimensionar correctamente el sistema de gestión de energía, reduciendo el tamaño de estos y a su vez el peso dentro del satélite.

Palabras clave: CubeSat, energía; paneles fotovoltaicos; nanosatélites; potencia eléctrica; energía eléctrica; MATLAB; gestión energética.

\begin{abstract}
A satellite is a device that is placed in orbit around the earth for scientific, military, educational, or communications purposes. They can be classified according to their weight as: large satellites weighing more than 1,000 kilograms (kg), medium satellites weighing between $500-1000 \mathrm{~kg}$, and small satellites, weighing less than $500 \mathrm{~kg}$. Within the small satellites are the nanosatellites, which weigh between 1-10 kg. The main advantage of nanosatellites is that they are capable of performing complex missions at a lower cost. Among the limitations that this present is that they have little capacity to capture and store energy due to their size. Considering the importance of the power system during the operation of satellites, this article analyzes the failure modes that occur in the system and how these affect energy generation and storage systems. For the simulation of these systems the minimum number of solar panels and batteries necessary for their correct operation was determined. The results obtained show that with this analysis it is possible to correctly size the energy management system, reducing the size of these and in turn the weight within the satellite.
\end{abstract}

Keywords: CubeSat; energy; photovoltaic panels; nanosatellites; electrical power; electrical energy; MATLAB; energy management. 


\section{Introducción}

Los CubeSats son nanosatélites de dimensiones estandarizadas, compuestos por una o más unidades $(\mathrm{U})$, que tienen un tamaño de $10 \mathrm{~cm}$ x $10 \mathrm{~cm}$ x $10 \mathrm{~cm}$ y una masa de hasta $1.33 \mathrm{~kg}$ (Abdullah et al., 2021). Por lo general, los CubesSat están compuestos por diferentes subsistemas, los cuales dependen de la misión que deba desarrollar, entre ellos se encuentran el sistema de energía eléctrica, comando y manejo de datos, control de orientación, estructura y telemetría, comando y rango, propulsión y control térmico (Nieto-Peroy \& Emami, 2019; Filippi et al., 2019).

La principal fuente de energía en el espacio es el Sol, es por esto que el uso de paneles fotovoltaicos complementados con baterías de alta eficiencia son los elementos usados para proporcionar energía continua al sistema (Akyildiz et al., 2019). La eficiencia en la captación de energía solar depende de la posición en la que se encuentre el CubeSat. El sistema de energía eléctrica (EPS, por sus siglas en inglés) es el responsable de gestionar la generación, almacenamiento, regulación y distribución de la energía eléctrica en el satélite (Gonzalez-Llorente, 2016). Por esta razón se hace esencial el uso de baterías para el almacenamiento de energía eléctrica y de esta forma poder complementar el abastecimiento de energía eléctrica del mismo (Acharya et al., 2019). Además, el EPS se encarga de la regulación de energía eléctrica para mantener la tensión y corriente eléctrica a niveles adecuados para que los dispositivos electrónicos del CubeSat, y también distribuye la alimentación de energía eléctrica a los demás subsistemas (Aung et al., 2020).

Un $29 \%$ de los CubeSats lanzados han informado problemas en órbita causados por el EPS (Bouwmeester et al., 2017), siendo el segundo sistema más contribuyente a fallas en la etapa inicial y final de la vida útil (Langer \& Bouwmeester, 2016). Los problemas están categorizados de la siguiente manera: mal dimensionamiento del EPS, problemas electrónicos, fallas electrónicas, degradación de paneles solares, problemas con la batería y fallos de las baterías (Bouwmeester et al., 2017). El EPS es considerado uno de los sistemas más crítico, ya que esto pueden afectar a otros sistemas (Faure et al., 2017; Kim et al., 2012; Ibrahim et al., 2020; Latachi 
et al., 2020). Aun con el uso de métodos como el de análisis de modos de fallas, efectos y criticidad (FMECA, por sus siglas en inglés) para evaluar las posibles fallas en el EPS, siempre existirán fallas que no se podrán evitar cuando el CubeSat esté en órbita (Menchinelli et al., 2018).

Dentro de los modos de fallos que presenta el FMECA se encuentran: falta de energía, corte de energía de larga duración, fallos de los paneles fotovoltaicos, limitación de potencia disponible, fallo de baterías, fallo de electrónica, pérdida de comunicación y fallo de elementos externos.

El objetivo de este artículo es presentar un análisis de los posibles fallos que pueden ocurrir en el EPS y cómo lograr un consumo de energía eficiente en función a la misión. Con este análisis se presentan recomendaciones que permiten al CubeSat continuar en funcionamiento en caso de fallar el sistema de generación de energía. La estructura del artículo es como sigue: en la sección 2 se expone la metodología utilizada para el estudio; en el apartado 3 se presenta el dimensionamiento del sistema de energía eléctrica de los CubeSats. En el acápite 4 se detallan las simulaciones del sistema de generación y del sistema de almacenamiento. En la sección 5 se evidencian los resultados obtenidos de la simulación y, finalmente, en el apartado 6 se presentan las conclusiones.

\section{Dimensionamiento del sistema de energía}

Para el dimensionamiento del sistema de energía se utilizó como referencia QuisqueyaSat 1 (QSAT1), que es un CubeSat que está siendo desarrollado por el Instituto Tecnológico de Santo Domingo, cuya misión principal será identificar algas sargazo en las inmediaciones del Caribe y la isla de La Española. Además, tendrá como misión secundaria medir el espesor de la nube de polvo del Sahara sobre la República Dominicana. Se espera que el QSAT1 tenga una vida útil estimada de un ańo y orbite a una altitud de $500 \mathrm{~km}$.

Para determinar la cantidad de energía generada por los paneles de un CubeSat y representar una aproximación de la curva característica de los paneles fotovoltaicos, se utilizó el modelo matemático diseñado 
por Gil Arias y Ortiz Rivera (2008). La siguiente ecuación relaciona la corriente y voltaje del panel solar:

$$
I(V)=\frac{I_{x}}{1-e^{-\frac{1}{b}}}\left(1-e^{\left(\frac{V}{b V x}-\frac{1}{b}\right)}\right)
$$

donde:

$\mathrm{I}(\mathrm{V})=$ Corriente en función del voltaje

$\mathrm{I}_{\mathrm{x}}=$ Corriente del panel

$\mathrm{b}=$ Constante característica del panel

$\mathrm{V}=$ Variable independiente de voltaje

$\mathrm{V}_{\mathrm{x}}=$ Voltaje del panel

Similarmente, la siguiente ecuación modela el voltaje del panel solar

$$
V_{x}=S \frac{E_{i}}{E_{i N}} T C_{v}\left(T-T_{N}\right)+S V_{\max }-S\left(V_{\max }-V_{\min }\right) e^{\frac{E_{i}}{E_{i N}} \ln \left|\frac{V_{\max }-V_{o c}}{V_{\max }-V_{\min }}\right|}
$$

donde:

$\mathrm{V}_{\mathrm{x}}=$ Voltaje del panel

$\mathrm{S}=$ Cantidad de paneles conectados en serie

$\mathrm{E}_{\mathrm{i}}=$ Irradiancia operativa

$\mathrm{E}_{\mathrm{in}}=$ Irradiancia del ambiente $\left(1000 \mathrm{w} / \mathrm{m}^{2}\right)$

$\mathrm{TC}_{\mathrm{v}}=$ Constante del coeficiente de voltaje del panel

$\mathrm{T}=$ Temperatura operativa

$\mathrm{T}_{\mathrm{n}}=$ Temperatura ambiente

$\mathrm{V}_{\max }=103 \%$ del voltaje de circuito abierto

$\mathrm{V}_{\min }=85 \%$ del voltaje de circuito abierto

$\mathrm{V}_{\mathrm{oc}}=$ Voltaje de circuito abierto 
La corriente del panel solar se determina a partir de:

$$
I_{x}=P \frac{E_{i}}{E_{i N}}\left(I_{s c}+T C_{i}\left(T-T_{N}\right)\right.
$$

$$
\begin{aligned}
& \text { donde } \\
& \mathrm{I}_{\mathrm{x}}=\text { Corriente del panel } \\
& \mathrm{P}=\text { Cantidad de Paneles solares conectados en paralelo } \\
& \mathrm{E}_{\mathrm{i}}=\text { Irradiancia operativa } \\
& \mathrm{E}_{\mathrm{iN}}=\text { Irradiancia del ambiente } \\
& \text { Isc = Corriente de corto circuito } \\
& \mathrm{TC}_{\mathrm{i}}=\text { Constante del coeficiente de corriente del panel } \\
& \mathrm{T}=\text { Temperatura operativa } \\
& \mathrm{T}_{\mathrm{N}}=\text { Temperatura del ambiente }
\end{aligned}
$$

El requerimiento de energía de un CubeSat se compone por la sumatoria del consumo de subsistemas activos y la potencia de carga útil (Arnold et al., 2012). La carga útil se refiere a los equipos que el satélite utiliza para ejecutar las tareas por las que fue puesto en órbita, y esta varía según los objetivos de cada satélite. Por lo regular, la potencia eléctrica demandada por los CubeSat ronda entre 1 y 10 watts (Carvalho et al., 2020).

El satélite se utilizará en dos misiones específicas; para la misión 1 , que es la identificación de algas sargazo, la potencia requerida es de 1.4325 W, mientras que para la misión 2, que es la medida del espesor de la nube de polvo del Sahara, la potencia requerida es de $1.4325 \mathrm{~W}$. En caso de realizar las dos misiones simultáneamente, la potencia requerida es de $1.5075 \mathrm{~W}$ y cuando no se está realizando ninguna misión la potencia requerida es de $1.3575 \mathrm{~W}$.

El EPS tiene dos arquitecturas que pueden seguirse para diseñar el sistema: arquitectura centralizada y distribuida (Mohammed et al., 2017). 
La principal ventaja que presenta la arquitectura centralizada es que utiliza un regulador para proporcionar la misma tensión de voltaje para múltiples subsistemas (Burt, 2011). La arquitectura distribuida posee la ventaja que tiene un convertidor de punto de carga responsable de regular su propio bus de nivel inferior voltajes (Burt, 2011). Debido a la ventaja que presenta la arquitectura distribuida fue la configuración seleccionada, ya que resulta ser más eficiente.

Con base en la configuración elegida, se seleccionó el sistema P31-U de la empresa GomSpace (GOMspace, 2020), el cual cumple con la configuración antes mencionada. El sistema $\mathrm{P} 31-\mathrm{U}$ incorpora paneles solares que están compuestos de celdas solares de triple unión de sustrato con una de eficiencia 30 \% (AZUR SPACE Solar Power GmbH, 2020). Debido a que una cara del CubeSat tiene un área de $100 \mathrm{~cm}^{2}$, se podrían ubicar máximo dos paneles por cara.

Para cargar las baterías existen dos métodos comúnmente utilizados, que son: transferencia directa de energía (DET) y seguimiento del punto de máxima potencia (MPPT) (Carvalho et al., 2020). Para este caso se seleccionó la configuración DET, la razón de la selección de este método se debe a permite simplificar la interconexión entre la celdas solares y el bus de voltaje permitiendo reducir las pérdidas resultado de las transferencia de energía (Lim et al., 2018). Este método consiste en una conexión directa entre los paneles y la batería mediante semiconductores de potencia.

\section{Simulación del sistema de gestión}

Para el análisis del comportamiento de los sistemas de generación y almacenamiento se evaluaron diferentes combinaciones. En la figura 1 se muestran los diferentes escenarios que se consideraron para la simulación. En cada uno de los casos se evaluó la generación de energía eléctrica por los paneles solares y el uso de las baterías. 


\section{Figura 1}

Diferentes escenarios para análisis de escenarios del comportamiento del EPS

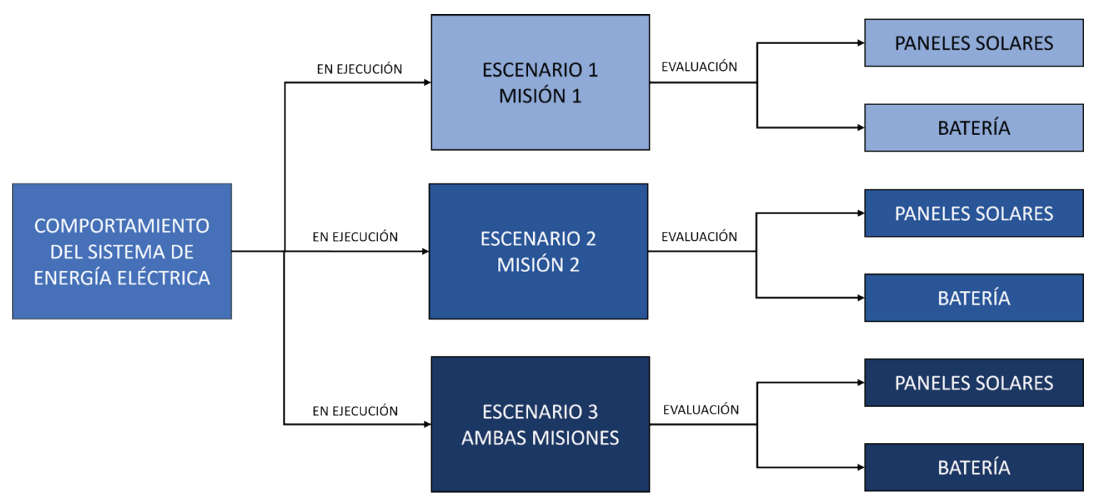

Para las simulaciones sobre generación de energía eléctrica se utilizó el procedimiento descrito por González Llorente y Puerto Leguizamón (2014), donde se estima la cantidad máxima de potencia que puede obtenerse de los paneles solares.

\subsection{Simulación Paneles}

Para la simulación de los paneles se construyeron los bloques que representan las ecuaciones para determinar el comportamiento del voltaje y la corriente de los paneles solares, como se puede apreciar en la figura 2 .

\section{Figura 2}

Simulación del comportamiento de los paneles
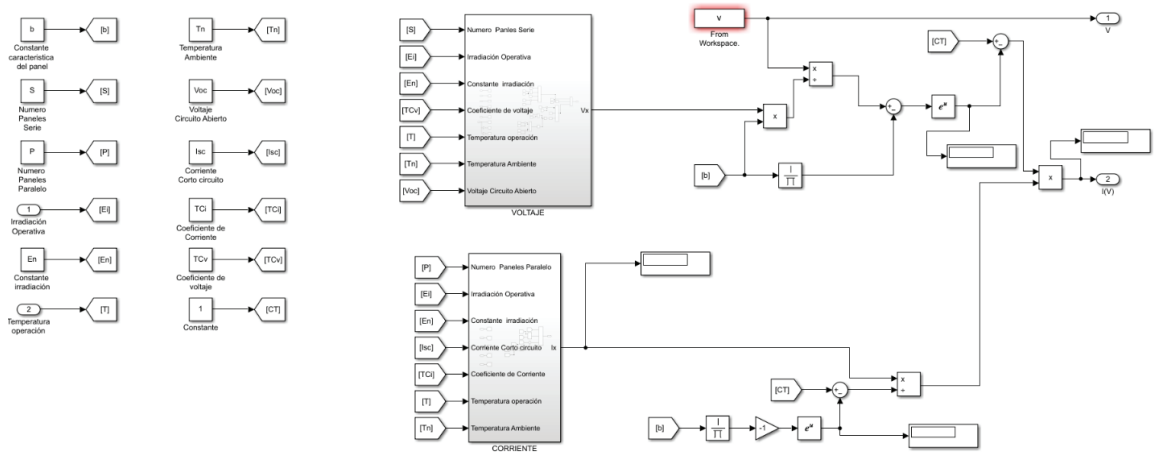
Para la simulación de los escenarios relacionados con las misiones, se construyeron los bloques correspondientes a los paneles solares (véase figura 3) donde se simuló el comportamiento del sistema en diferentes escenarios.

\section{Figura 3}

Bloques de simulación de paneles para los diferentes escenarios

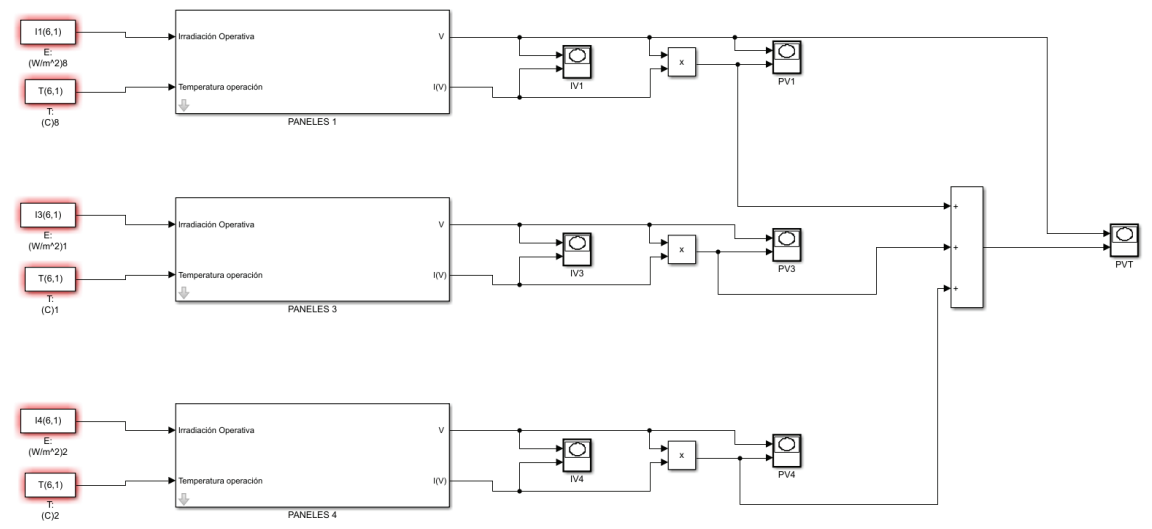

En la tabla 1 se muestran los datos utilizados para la caracterización de los paneles. Estos datos fueron obtenidos de la hoja de datos del fabricante de los paneles vía (GOMspace, 2020).

\section{Tabla 1}

Datos técnicos de los paneles solares según el fabricante

\begin{tabular}{lc}
\hline Datos & Valores \\
\hline Constante característica del panel & 0.0684 \\
\hline Número de paneles en serie & 2 \\
\hline Número de paneles en paralelo & 1 \\
\hline Constante de irradiación $\left(\mathrm{W} / \mathrm{m}^{2}\right)$ & 1367 \\
\hline Temperatura del ambiente $\left({ }^{\circ} \mathrm{C}\right)$ & 28 \\
\hline Voltaje de circuito abierto $(\mathrm{V})$ & 2.69 \\
\hline Coeficiente de corriente $(\mathrm{A})$ & 0.00036 \\
\hline Coeficiente de voltaje & -0.0062 \\
\hline
\end{tabular}


Cuando se ejecutaron las simulaciones se consideró que el satélite tendría una altitud de $500 \mathrm{~km}$ y el tiempo que tardaría en darle una vuelta a la Tierra según (González Llorente \& Puerto Leguizamón, 2014) sería de 99 minutos, aprovechando la luz solar el $66.43 \%$ del tiempo. Adicional, se consideró un caso extremo donde solo el $50 \%$ del tiempo, equivalente a 49.5 minutos, estaría aprovechando la luz solar.

\subsection{Simulación de baterías}

Para la simulación de los estados de carga y descarga de las baterías, como se puede ver en la figura 4, se utilizó un bloque de batería que viene integrado en Simulink, en el cual se obtiene el porcentaje de estado de carga de la batería (SOC, por sus siglas en inglés). Considerando que el consumo máximo del CubeSat en un día de misión es de $32.93 \mathrm{Wh}$, se obtuvo que la batería requerida para el CubeSat tendría las siguientes especificaciones: 8 celdas, 77 Wh de capacidad, rango de 6-8.4 voltios, voltaje nominal de $7.4 \mathrm{~V}$ y cantidad de carga eléctrica de $10 \mathrm{Ah}$. Adicionalmente, se determinó que el punto mínimo de descarga sería de un $50 \%$.

\section{Figura 4}

Bloques de simulación de carga y descarga de las baterías

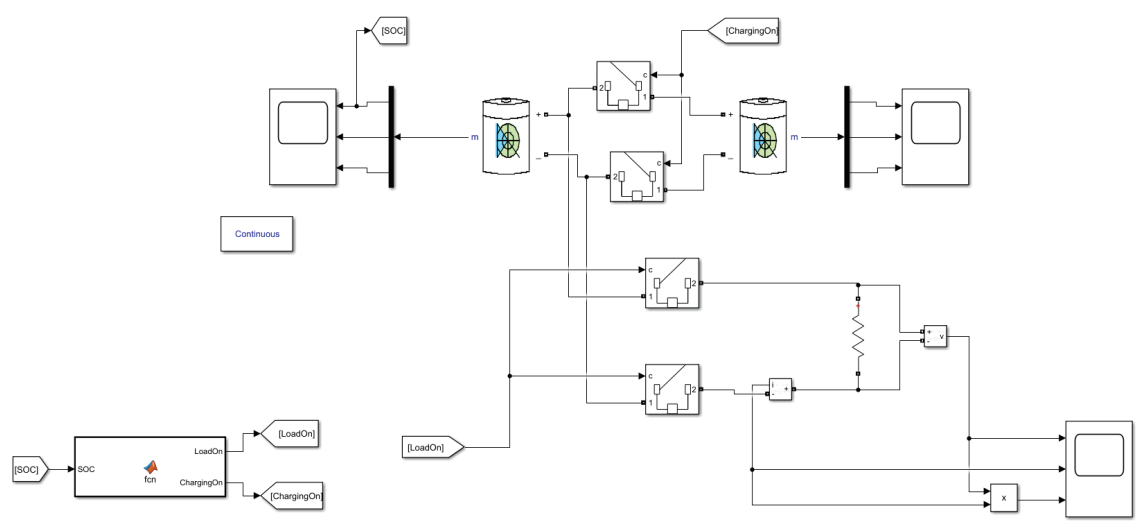




\section{Discusión}

4.1 Resultados de simulación de paneles solares

\subsubsection{Condición normal}

Se determinó que la potencia puede alcanzar un valor de $1.4325 \mathrm{~W}$, la cual puede ser cubierta por la batería el $50.87 \%$ del tiempo y el $49.13 \%$ restante sería cubierto por los paneles solares.

\subsubsection{Falla en una cara}

En la tabla 2 se muestran las condiciones para los escenarios de que falle una sola cara, siendo el escenario más crítico cuando falla la cara 3 debido a que habrá más dependencia del sistema de almacenamiento.

\section{Tabla 2}

Resultados de las simulaciones cuando falla una cara de paneles solares

\begin{tabular}{lccc}
\hline Demanda Cubierta & Cara 1 & Cara 3 & Cara 4 \\
\hline Batería (\%) & 60.90 & 70.20 & 66.09 \\
\hline Paneles Solares (\%) & 39.10 & 29.80 & 33.91 \\
\hline
\end{tabular}

\subsubsection{Falla en dos caras}

En la tabla 3 se muestran las condiciones en caso de que fallen dos caras, siendo el caso más crítico cuando fallan los paneles solares de las caras 3 y 4, debido a que, igual que en el escenario anterior, habrá más dependencia del sistema de almacenamiento.

\section{Tabla 3}

Resultados de las simulaciones cuando falla una cara de paneles solares

\begin{tabular}{lccc}
\hline Demanda Cubierta & Cara 1 y 3 & Cara 1 y 4 & Cara 3 y 4 \\
\hline Batería (\%) & 80.97 & 91.31 & 84.78 \\
\hline Paneles Solares (\%) & 19.03 & 18.69 & 15.22 \\
\hline
\end{tabular}


4.1.4 Falla en todos los paneles solares

Cuando fallan todos los paneles solares la potencia generada es completamente cero, por lo que toda la demanda debe suplirse por parte de las baterías, la cual solo podría hacerlo por un tiempo de 24 horas.

4.2 Resultados de simulación de carga y descarga de baterías

\subsubsection{Condición normal}

Dado el comportamiento de la batería a nivel promedio, la carga aumentaría $1.73 \%$ cada vez que pasa por un estado de carga, y disminuiría un $1.42 \%$ cuando estuviera siendo utilizada.

\subsubsection{Falla en una cara}

La tabla 4 muestra, en caso de fallar una cara, cuál sería el comportamiento de carga y descarga, siendo el caso más crítico cuando falla la cara 3.

\section{Tabla 4}

Resultados de las simulaciones del comportamiento de la batería cuando falla una cara

Nivel Promedio $\quad$ Cara $1 \quad$ Cara $3 \quad$ Cara 4

Carga de batería (\%)

1.44

0.77

Descarga de batería (\%)

1.02

1.82

0.89

\subsubsection{Falla en dos caras}

Para este caso, como se puede ver en la tabla 5, el más crítico es cuando la cara 4 se encuentra entre las dos caras que fallan. 


\section{Tabla 5}

Resultados de las simulaciones del comportamiento de la batería cuando fallan dos caras

\begin{tabular}{lcll}
\hline Nivel Promedio & Cara 1 y 3 & Cara 1 y 4 & Cara 3 y 4 \\
\hline Carga de batería (\%) & 1.44 & 1.57 & 1.57 \\
\hline Descarga de batería (\%) & 1.88 & 2.03 & 2.03 \\
\hline
\end{tabular}

4.2.4 Falla en todos los paneles solares

Al producirse el fallo de todos los paneles solares, el nivel promedio en que disminuye la batería sería de $22.48 \%$.

\section{Conclusión}

En este artículo se analizó los modos fallas en el sistema de gestión de energía de un nanosatélite tipo CubeSat a través de modelos matemáticos implementados en el software MATLAB/Simulink describiendo el comportamiento del sistema de generación y el sistema de almacenamiento. A través de las simulaciones se analizaron diferentes fallas, considerando diferentes posibles escenarios. Los resultados muestran que dependiendo del número de caras que presenten fallas el CubeSat puede continuar funcionando de manera adecuada.

En caso de fallar una cara este podrá seguir operando, teniendo en cuenta que las baterías estarían consumiendo entre un $10 \%$ a $20 \%$ por encima de la demanda en condición normal. De fallar dos caras, las baterías tendrían un consumo por encima de la demanda en condición normal de un $20 \%$ a $30 \%$ implicando que su carga sería más lenta. En caso de fallar las tres caras, la batería solo otorgaría una autonomía de un día. Si fallara el módulo de batería, el satélite no sería capaz de cumplir con su objetivo, debido a que durante el tiempo que los paneles solares no reciben radiación solar este se encuentra fuera de servicio. Dentro de futuras líneas de investigación se hace la propuesta del análisis de un sistema operativo de tiempo real (RTO, por sus siglas en inglés) para mejorar la gestión del sistema de gestión de energía del nanosatélite. 


\section{Agradecimientos}

Esta investigación fue apoyada por el Ministerio Educación Superior Ciencia y Tecnología (MESCyT) de la República Dominicana a través del programa FONDOCYT 2018-1H1-IN-00.1

\section{Referencias}

Abdullah, H. H., Elboushi, A., Gohar, A. E., \& Abdallah, E. A. (2021). An Improved S-Band CubeSat Communication Subsystem Design and Implementation. IEEE Access, 9, 45123-45136. https://doi. org/10.1109/ACCESS.2021.3066464

Acharya, S., Alshehhi, F., Tsoupos, A., Khan, O., Elmoursi, M., Khadkikar, V., Zeineldin, H., \& Al Hosani, M. (2019). Modeling and Design of Electrical Power Subsystem for CubeSats. 2019 International Conference on Smart Energy Systems and Technologies (SEST), 1-6. https://doi.org/10.1109/SEST.2019.8849042

Akyildiz, I. F., Jornet, J. M., \& Nie, S. (2019). A new CubeSat design with reconfigurable multi-band radios for dynamic spectrum satellite communication networks. Ad Hoc Networks, 86, 166-178. https://doi.org/https://doi.org/10.1016/j.adhoc.2018.12.004

Arnold, S. S., Nuzzaci, R., \& Gordon-Ross, A. (2012). Energy budgeting for CubeSats with an integrated FPGA. 2012 IEEE Aerospace Conference, 1-14. https://doi.org/10.1109/AERO.2012.6187240

Aung, H., Soon, J. J., Goh, S. T., Lew, J. M., \& Low, K.-S. (2020). Battery Management System With State-of-Charge and Opportunistic Stateof-Health for a Miniaturized Satellite. IEEE Transactions on Aerospace and Electronic Systems, 56(4), 2978-2989. https://doi.org/10.1109/ TAES.2019.2958161

AZUR SPACE Solar Power GmbH. (2020). Triple Junction GaAs Solar Cell Assembly Type: TJ Solar Cell Assembly 3G30A. http://www.azurspace. $\mathrm{com} /$ index.php/en/products/products-space/space-assemblies

Bouwmeester, J., Langer, M., \& Gill, E. (2017). Survey on the implementation and reliability of CubeSat electrical bus interfaces. CEAS Space Journal, 9(2), 163-173. https://doi.org/10.1007/ s12567-016-0138-0 
Burt, R. (2011). Distributed Electrical Power System in Cubesat Applications [Utah State University]. https://digitalcommons.usu.edu/cgi/viewcontent.cgi?article $=2070 \&$ context $=$ etd

Carvalho, R. A. de, Estela, J., \& Langer, M. (2020). Nanosatellites: Space and Ground Technologies, Operations and Economics (John Wiley \& Sons (ed.)). https://www.wiley.com/en-ag/Nanosatellites\%3A+Space+and+Ground + Technologies $\% 2 \mathrm{C}+$ Operations+and+Economics+-p-9781119042037

Faure, P., Tanaka, A., \& Cho, M. (2017). Toward lean satellites reliability improvement using HORYU-IV project as case study. Acta Astronautica, 133, 33-49. https://doi.org/https://doi.org/10.1016/j. actaastro.2016.12.030

Filippi, G., Vasile, M., Krpelik, D., Korondi, P. Z., Marchi, M., \& Poloni, C. (2019). Space systems resilience optimisation under epistemic uncertainty. Acta Astronautica, 165, 195-210. https://doi.org/ https://doi.org/10.1016/j.actaastro.2019.08.024

Gil-Arias, O., \& Ortiz-Rivera, E. I. (2008). A general purpose tool for simulating the behavior of PV solar cells, modules and arrays. 2008 11th Workshop on Control and Modeling for Power Electronics, 1-5. https://doi.org/10.1109/COMPEL.2008.4634686

GOMspace. (2020). NanoPower P110. https://gomspace.com/UserFiles/ Subsystems/datasheet/gs-ds-nanopower-p110-210.pdf

Gonzalez-llorente, J. (2016). Análisis de la Capacidad de Almacenamiento de las Baterias para un Satélite CubeSat 3U. July.

González Llorente, J. D., \& Puerto Leguizamón, G. A. (2014). Estimación de la cantidad de potencia suministrada por las celdas fotovoltaicas de un cubesat. Revista Tecnura, 18(41), 53. https://doi.org/10.14483/ udistrital.jour.tecnura.2014.3.a04

Kim, S. Y., Castet, J.-F., \& Saleh, J. H. (2012). Spacecraft electrical power subsystem: Failure behavior, reliability, and multi-state failure analyses. Reliability Engineering \& System Safety, 98(1), 55-65. https://doi.org/ https://doi.org/10.1016/j.ress.2011.10.005

Langer, M., \& Bouwmeester, J. (2016). Reliability of CubeSats - Statistical Data, Developers' Beliefs and the Way Forward. Small Satellite Conference. https://digitalcommons.usu.edu/smallsat/2016/ TS10AdvTech $2 / 4$ 
Lim, T. M., Cramer, A. M., Lumpp, J. E., \& Rawashdeh, S. A. (2018). A Modular Electrical Power System Architecture for Small Spacecraft. IEEE Transactions on Aerospace and Electronic Systems, 54(4), 1832-1849. https://doi.org/10.1109/TAES.2018.2803598

Menchinelli, A., Ingiosi, F., Pamphili, L., Marzioli, P., Patriarca, R., Costantino, F., Piergentili, F., Monteiro, J. P., Rocha, R. M., Silva, A., Afonso, R., Ramos, N., Stesina, F., Corpino, S., Latachi, I., Rachidi, T., Karim, M., Hanafi, A., Park, Y.-K., ... Park, S.-Y. (2018). A Reliability Engineering Approach for Managing Risks in CubeSats. In Aerospace (Vol. 5, Issue 4). https://doi.org/10.3390/ aerospace 5040121

Mohammed Abaker, A. A., \& Abd AlNabi Mustaf, A. B. (2017). Analysis of Cube-Sat Electrical Power System Architecture. Science Technology \& Innovation Journal (STIJ), 1(1), 1-6. https://techhorizon.fu.edu. sd/wp-content/uploads/2018/02/arafa_p2-1.pdf

Nieto-Peroy, C., \& Emami, M. R. (2019). CubeSat Mission: From Design to Operation. Applied Sciences, 9(15). https://doi.org/10.3390/ app9153110 